\title{
Application of the Cosine Gray Model Based on System Cloud in the Forecast of Higher Education scale
}

\author{
http://dx.doi.org/10.3991/ijet.v10i8.5207 \\ Chao Ge, Cun Li \\ Agricultural University of Hebei Baoding, Hebei, China
}

\begin{abstract}
The scale of higher education is an essential link in the process of the formulation of education planning and reasonable allocation of teaching resources. At the same time, it also provides the required basis and support for the government to formulate educational planning and policy. The scale of higher education development is influenced not only by the level of economic development and industrial structure, but also by the total population and the living standards of residents. We take these elements as the influence factors, which contain noise information. Because the scale of higher education and its impact factors have complex nonlinear relationship, the traditional forecasting method cannot describe their changing trends, which leads to the low accuracy of prediction. In order to solve the above problems, this paper bases on the traditional GM $(1,1)$ model to judge the number of students in the future, and uses the weakening buffer operator to amend the historical data. Secondly, this paper analyzes the structure of the system cloud gray forecasting model, and demonstrates its integral generation principle. We propose a new method for the cosine gray forecasting model which is based on the system cloud $\square$ SCOS-GM $(1,1)$, and prove the effectiveness of $\operatorname{SCOS-GM}(1,1)$ model by the residual test. Finally, the SCOS-GM $(1,1)$ model is utilized to predict the scale of higher education in China during the period of 2012-2014. The results show that the scale of higher education will demonstrate a gradual upward trend in the next few years.
\end{abstract}

Index Terms-system cloud, higher education scale, gray model

\section{INTRODUCTION}

The scale of higher education is a key link in the process of the formulation of education planning and reasonable allocation of teaching resources. At the same time, it also provides the necessary basis and support for the government to formulate educational planning and policy. The scale of higher education development is influenced not only by the level of economic development and industrial structure, but also by the total population and the living standards of residents. In addition, the cultural environment and the national higher education decisionmaking will generate a certain impact on the scale of higher education. Some of these factors are deterministic, while some are uncertain. In practice, the amount of data is small, which leads to difficulty of predicting the scale of higher education.

The scale of higher education is the product of the interaction between demand and supply [1-3]. Demand is divided into social needs and personal needs. It includes the population, the country's economic development, em- ployment absorption capacity, scientific and technological progress, the demand for higher education, and the living standards. Supply is mainly the conditions for running a school, especially the scale of teachers, the living standards of residents, etc. Among them, some of the factors both have the demand and supply factors, such as population, economic development, living standards of residents and so on[4-6]. As everyone knows, GDP is the most important indicator of a country's economic development. This factor can affect the demand for higher education, and it is the basis of the supply of higher education. Both domestic and foreign studies have indicated that the higher education gross enrollment rate or higher education scale have a certain degree of positive correlation with GDP[7-9]. Yue Changjun using the regression method shows that the proportion of higher education and GDP per capita has a linear relationship [10]. Another factor is the employment, which is supplied by the economic development in the future. This factor can be seen as an element affecting the demand for higher education. Domestic scholars have proved that the scale of higher education is closely related to the third industry, but the relationship with the first and the second industry is not close [11-13].

To sum up, we take these factors as the influence factors, which contain noise information. Because the scale of higher education and its influence factors have complex nonlinear relationship, the traditional forecasting method cannot describe their changing trends, which leads to the low accuracy of prediction. In order to solve the above problems, this paper bases on the traditional GM $(1,1)$ model to judge the number of students in the future, and uses the weakening buffer operator to amend the historical data. Secondly, this paper analyzes the structure of the system cloud gray forecasting model, and demonstrates its integral generation principle. We propose a new method for the cosine gray forecasting model which is based on the system cloud (SCOS-GM $(1,1)$, and prove the effectiveness of SCOS-GM $(1,1)$ model by the residual test. Finally, the SCOS-GM $(1,1)$ model is used to predict the scale of higher education in China during the period of 2012-2014. The results show that the scale of higher education will demonstrate a gradual upward trend in the next few years. The structure of this paper is as follows. We introduce the preliminary knowledge of the grey model in the second section; In the section 3 , the forecasting process of the traditional grey model is given; In the sections 4 and 5 , we construct the cosine gray prediction model based on the system cloud, and use the model to forecast the scale of higher education in China; The last two sections are conclusions and references. 


\section{RELATED WORK}

Grey system theory was a kind of data processing method which was built up in the last century. It was a method of data processing to deal with partially unknown information.

First, we introduce some basic knowledge of grey theory.

\section{A. Accumulating generation operator}

We assume that the initial sequence is:

$X^{(0)}=\left\{x^{(0)}(1), x^{(0)}(2), \mathrm{L} x^{(0)}(n)\right\}$

Where, $n$ is the dimension, $X^{(0)}$ is the grey sequence, and the $n \geq 1, x^{(0)}(i) \geq 0$

We define the new sequence as shown below

$X^{(1)}=\left\{x^{(1)}(1), x^{(1)}(2), \mathrm{L} x^{(1)}(n)\right\}$

Among them

$$
x^{(1)}(k)=\sum_{i=1}^{k} x^{(0)}(i)
$$

$X^{(1)}$ is a positive cumulative generating sequence of $X^{(0)}$,

Namely $X^{(1)}=A G O X^{(0)}$

If we carry on the $r$ th cumulative operation on the basis of the formula (1), we can get

$$
\text { If } x^{(r)}(k)=\sum_{i=1}^{k} x^{(r-1)}(i) \quad x^{(0)}=x^{(1)}(k)-x^{(1)} \cdot(k-1)
$$

Then, $X^{(1)}$ is a positive subtraction generating sequence of $X^{(0)}$,

Namely

$$
X^{(1)}=I A G O X^{(0)}
$$

If we carry on the $r$ th subtraction operation on the basis of the formula (1), we can get

$$
x^{(r-1)}=x^{(r)}(k)-x^{(r)} \cdot(k-1)
$$

\section{B. Inverse accumulating generation operator}

We assume the $X^{(0)}=\left\{x^{(0)}(1), x^{(0)}(2), \mathrm{L} x^{(0)}(n)\right\}$ is $n$ dimensional nonnegative decreasing sequence:

$$
x^{(0)}(i) \geq x^{(0)}(i+1) \quad i=1,2, \mathrm{~L}, n-1
$$

We define the new sequence as shown below

$$
X^{(1)}=\left\{x^{(1)}(1), x^{(1)}(2), \mathrm{L} x^{(1)}(n)\right\}
$$

Among them

$$
x^{(1)}(k)=\sum_{i=n}^{k} x^{(0)}(i)
$$

The sequence is the inverse accumulating generation operator

$$
x^{(0)}=x^{(1)}(k)-x^{(1)} \cdot(k+1)
$$

Formula (3) is the reduction formula of Formula (2).

\section{The Traditional GM $(1,1)$ Model}

Set the non-negative sequence is

$$
x^{(0)}=\left\{x^{(0)}(1), x^{(0)}(2), \mathrm{L}, x^{(0)}(n)\right\}
$$

Then the first-order accumulated generating operation sequence is

$$
x^{(1)}=\left\{x^{(1)}(1), x^{(1)}(2), \mathrm{L}, x^{(1)}(n)\right\}
$$

Which $x^{(1)}(k)=\sum_{i=1}^{k} x^{(0)}(i)$

Let the background value series $b$

$z^{(1)}(\mathrm{k})=\frac{1}{2}\left[x^{(1)}(k-1)+x^{(1)}(k)\right] k=2,3, \mathrm{~L}, \mathrm{n}$,

The grey differential equation is constructed with $x^{(1)}$ by Eq. (4)

$$
\frac{d x^{(1)}}{d t}+a x^{(1)}=u
$$

Which $a$ is called development coefficient. $u$ is called grey action variable.

The discrete form of Eq. (7) is

$$
x^{(0)}(\mathrm{k})+a z^{(1)}(\mathrm{k})=u
$$

Since

$$
\frac{\Delta x}{\Delta t}=\frac{x^{(1)}(k+1)-x^{(1)}(k)}{k+1-k}=x^{(1)}(k+1)-x^{(1)}(k)=x^{(0)}(k+1)
$$

The matrix expression of $\mathrm{GM}(1,1)$ is

$$
Y_{n}=B A
$$

$$
Y_{n}=\left(\begin{array}{c}
x^{(0)}(2) \\
x^{(0)}(3) \\
\mathrm{M} \\
x^{(0)}(n)
\end{array}\right), A=\left(\begin{array}{l}
a \\
u
\end{array}\right), B=\left(\begin{array}{ll}
-z^{(1)}(2) & 1 \\
-z^{(1)}(3) & 1 \\
\mathrm{M} & \mathrm{M} \\
-z^{(1)}(n) & 1
\end{array}\right)
$$

The parameters is solved by least squares method

$$
\hat{A}=\left(\begin{array}{l}
\hat{a} \\
\hat{u}
\end{array}\right)=\left(B^{T} B\right)^{-1} B^{T} Y_{n}
$$

Put parameters into grey differential equation (4), the solution is

$$
x^{(1)}(t)=\left[x^{(1)}(1)-\frac{\hat{u}}{\hat{a}}\right] e^{-\hat{a} t}+\frac{\hat{u}}{\hat{a}}
$$

The discrete form of solution is

$$
\begin{gathered}
x^{(1)}(k+1)=\left[x^{(0)}(1)-\frac{\hat{u}}{\hat{a}}\right] e^{-\hat{a} k}+\frac{\hat{u}}{\hat{a}} \\
k=1,2, \mathrm{~L} n
\end{gathered}
$$

Restore the first-order accumulated generating operation; we will get the prediction form.

$\hat{x}^{(0)}(k+1)=\hat{x}^{(1)}(k+1)-\hat{x}^{(1)}(k)=\left(1-e^{\hat{a}}\right)\left(x^{(0)}(1)-\frac{\hat{u}}{\hat{a}}\right) e^{-\hat{a} k}$
$k=1,2, \mathrm{~L}, n$

\section{The System Cloud Cosine Gray Model}

Proportion of smooth discrete function are very important to grey model. The smoothness optimization of initial sequence is the effective method to improve the accuracy of grey model. In this paper, the cosine transform is used to improve the smoothness of the original sequence. According to the actual situation of university education, the total amount of students is the annual data, 
the original data sample is less and the statistical information is not perfect. So, it is difficult to predict the scale of higher education with the general mathematical statistics method. The theoretical foundation of the cosine gray prediction model based on system cloud (SCOS-GM (1, $1)$ ) is more reliable. It has the characteristics of less information, simple calculation, and higher accuracy. This method does not need to consider the complex factors of the college education. It can find the inherent law from its own sequence. So the cosine gray model based on system cloud is an ideal model to predict the number of university students.

The original time series of the number of university students is $Y^{(0)}(k)$

$$
Y^{(0)}(k)=\left\{y^{(0)}(1), y^{(0)}(2), \mathrm{L}, y^{(0)}(\mathrm{n})\right\}
$$

Which $y^{(0)}(\mathrm{i}) \in\left(0, \frac{\pi}{2}\right) i=1,2, \mathrm{~L}$

Then, the sequence is processed by cosine transform, that is

$$
X^{(0)}=\left\{x^{(0)}(1), x^{(0)}(2), \mathrm{L}, x^{(0)}(n)\right\}
$$

Which $x^{(0)}(k)=\cos y^{(0)}(k), i=1,2, \mathrm{~L}$

Then the first-order accumulated generating operation sequence is

$$
X^{(1)}=\left\{x^{(1)}(1), x^{(1)}(2), \mathrm{L}, x^{(1)}(n)\right\}
$$

Which $\quad x^{(1)}(k)=\sum_{i=1}^{k} x^{(0)}(i) \quad k=1,2, \mathrm{~L}$

In this paper, we assume the integral generating sequence $\left\{\hat{X}^{(1)}(k)\right\}$ of the number of university students and non-homogeneous exponential dispersion function $f_{r}(k)=b e^{a(k-1)}-c$ meet the trend relation. Then, the SCOS-GM $(1,1)$ model is:

$$
\frac{d X^{(1)}(k)}{d k}=a X^{(1)}(k)+U, \quad k \geq 2
$$

Its response function is:

$$
\hat{X}^{(1)}(k)=\left(\hat{X}^{(1)}(1)+\frac{U}{a}\right) e^{a k}-\frac{U}{a}
$$

In the formula (19):

$$
\begin{gathered}
a=\ln \frac{\sum_{k=3}^{n} \hat{X}^{(0)}(k-1) \hat{X}^{(0)}(k)}{\sum_{k=3}^{n}\left(\hat{X}^{(0)}(k-1)\right)^{2}} \\
b=\frac{(n-1) \sum_{k=2}^{n} e^{a(k-1)} \hat{X}^{(1)}(k)-\sum_{k=2}^{n} e^{a(k-1)} \sum_{k=2}^{n} \hat{X}^{(1)}(k)}{(n-1) \sum_{k=2}^{n} e^{2 a(k-1)}-\sum_{k=2}^{n} e^{a(k-1)}} \\
c=\frac{1}{n-1}\left[b \sum_{k=2}^{n} e^{a(k-1)}-\sum_{k=2}^{n} \hat{X}^{(1)}(k)\right]
\end{gathered}
$$

Which, $\hat{X}^{(1)}(1)=b-c, U=a c$

Reduction $\left\{\hat{X}^{(1)}(k)\right\}$, we get the SCOS-GM $(1,1)$ prediction model is

$$
\hat{X}^{(0)}(k)=\frac{2 b\left(1-e^{-a}\right)}{\left(1+e^{-a}\right)} e^{a(k-1)}
$$

Set

$$
Y(k)=\frac{X^{(0)}(k)}{\hat{X}^{(0)}(k)}
$$

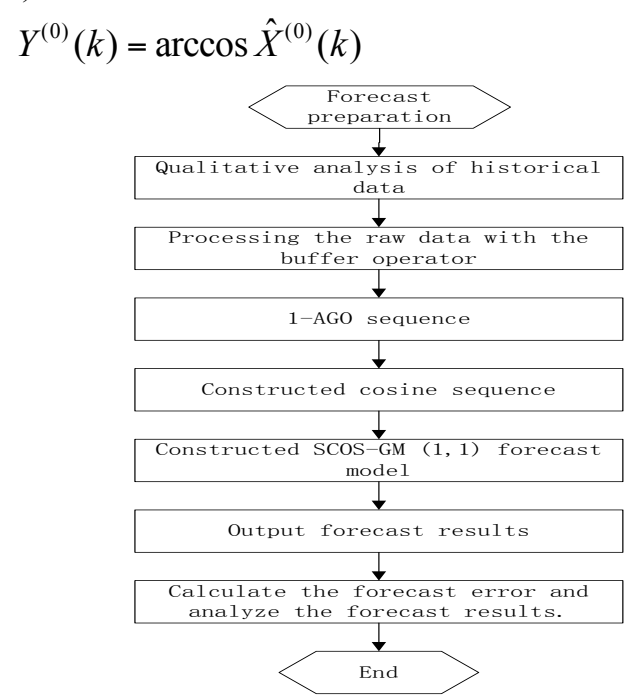

Figure 1. Prediction procedure of combinative improved grey model

\section{EXPERIMENT AND RESULT ANALYSIS}

Using the SCOS-GM $(1,1)$ model to forecast the scale of higher education in China. There are many indicators to measure the scale of higher education, such as the higher education enrollment rate, the number of college students, etc. The higher education enrollment rate reflects the degree of difficulty of the school-age youth enrollment. It can't reflect the scale of higher education in our country very well. The number of students in universities can be reflected in the size of the buildings, which are mainly used in study the living condition of students. In addition, it also reflects the scale of teachers' team, which is equipped with a precise ratio of teachers and students in the universities. So it reflect the overall strength of universities. In this paper, the original data set of the prediction model is constructed with the number of college students in 2001-2011.This paper uses the SCOS-GM(1,1)model to forecast the scale of higher education in our country during the period of 2012-2014. The original data from the "China Statistical Yearbook 2011 Edition". The original sequence is illustrated in Figure 2.

Next, we use the mathematical model which is established in the section 4 of this article to predict the higher education scale in our country. The forecast results are as follows. First, according to the formula 26 and 27, we give a comparison of the prediction errors. From Table 1, we can find that the SCOS-GM $(1,1)$ model is better than the original GM $(1,1)$ model and artificial neural network (ANN) model on RMSE and MAE. It means that the SCOS-GM $(1,1)$ method of grey model is effective, and it is also has better performance than the ANN model in a certain situation.

The prediction values of different models in this paper are shown in Table 2. In practice, the total amount of our 


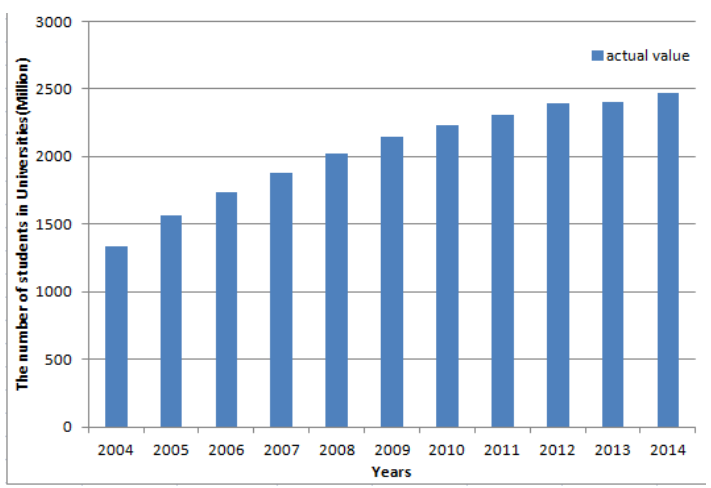

Figure 2. the number of students in university in China

TABLE I. THE PERFORMANCE OF DIFFERENT MODELS

\begin{tabular}{llll}
\hline & SCOS-GM $(1,1)$ & GM $(1,1)$ & ANN \\
\hline RMSE & 0.019 & 0.024 & 0.022 \\
MAE & 0.045 & 0.057 & 0.048 \\
\hline
\end{tabular}

Which

$$
\begin{aligned}
& R M S E=\sqrt{\frac{1}{n} \sum_{i=1}^{n}\left(\hat{\mathrm{y}}_{i}-\mathrm{y}_{i}\right)^{2}} \\
& M A E=\frac{1}{n} \sum_{i=1}^{n}\left|\frac{\hat{y}_{i}-y_{i}}{y_{i}}\right|
\end{aligned}
$$

country's graduates gradually expanded. At the same time, the structure problem of human resources in the enterprise is very obvious, and the graduates' employment ideas need to be further changed. We need to get rid of the barriers of institutional mechanisms for the employment of college graduates, deepen the comprehensive reform of education, enhance the adaptability of higher education, and improve the employment policy system, service system and market system.

Figure 4 gives the absolute error of different models on predicting number of students in university of our country from 2012 to 2014. From figure 4, the absolute error of optimized GM $(1,1)$ model is less than the other models in each year. The absolute errors are almost in the range of $6 \%$. We can easily find that the optimized model proposed in this paper is the best model compared with other models on absolute error.

\section{CONCLUSION}

This paper bases on the traditional GM $(1,1)$ model to judge the number of students in the future, and uses the weakening buffer operator to amend the historical data. Secondly, this paper analyzes the structure of the system cloud gray forecasting model, and demonstrates its integral generation principle. We propose a new method for the cosine gray forecasting model which is based on the system cloud SCOS-GM $(1,1)$, and prove the validity of SCOS-GM $(1,1)$ model by the residual test. Finally, the SCOS-GM $(1,1)$ model is used to predict the scale of higher education in China during the period of 20122014. Experimental results show that the proposed method has the ability of removing noise and high prediction accuracy. As we can see, the scale of higher education in China will increase year by year. The conclusion of this paper has certain guiding significance to the college enrollment, the proportion of the teaching facilities, and the teaching environment.

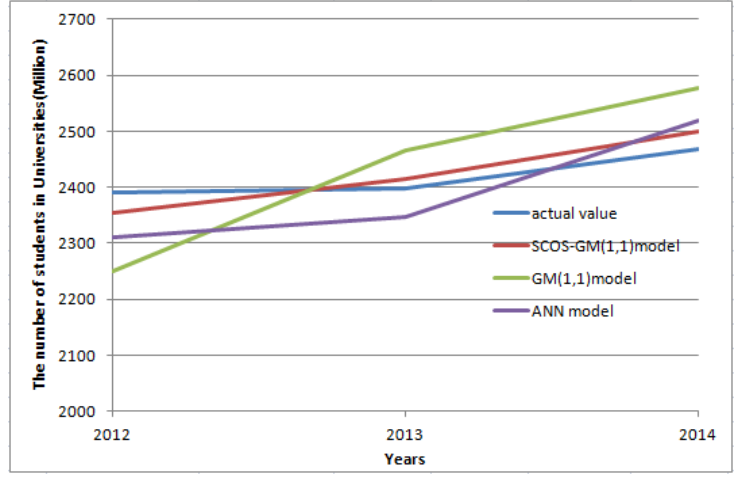

Figure 3. The prediction curve of Optimized GM(1,1) model

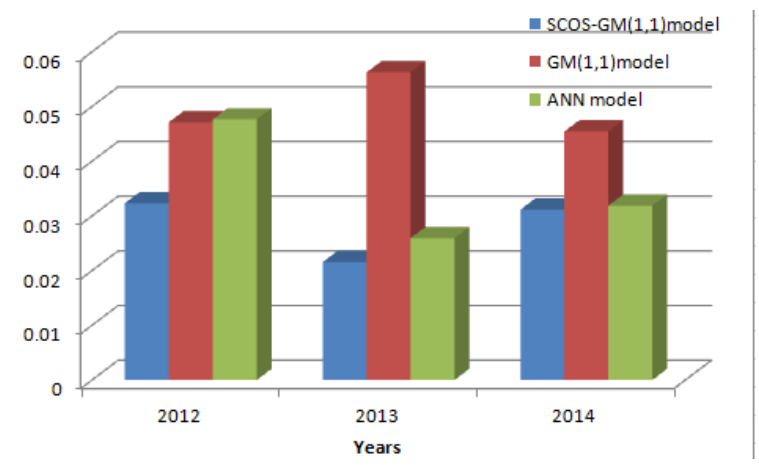

Figure 4. The absolute error of different models

\section{REFERENCES}

[1] Chen Liwen. Research on the appropriate scale of higher education in Hebei province [J]. Science and technology management, 2006, (2).

[2] BLEAUB L. Planning models in higher education: historical review and survey of currently available models $[\mathrm{J}]$. Higher Education, 1981, 10(2):153-168. http://dx.doi.org/10.1007/BF001 29129

[3] STINCH COMB H G. An application of student enrollment project ion models: to Georgia suburban school districts [D]. Athens, Georgia, 1985.

[4] BRAZZIEL W F. Forecasting older student enrollment: a cohort and participation rate model [J]. Journal of Higher Education, 1987, 58 (2): 223- 231. http://dx.doi.org/10.2307/1981243

[5] SALLEY C D. Short-term enrollment forecasting for accurate budget planning [J]. Journal of Higher Education, 1979, 50(3):323-333. http://dx.doi.org/10.2307/1980660

[6] CALVIN F S, SH ANLEY F J. Techniques in forecasting university enrollment [J]. Journal of Higher Education, 1952, 23 (9): 483- 488. http://dx.doi.org/10.2307/1976933

[7] Yi Weiping. The reasonable scale of higher education in our country from the international comparison $[\mathrm{J}]$. The research of education development, 2000, (2)

[8] T AN J P, MINGAT A. Education in Asia: A Comparative Study of Cost and Financing [M] .Washington, D.C: The World Bank, 1992. http://dx.doi.org/10.1596/0-8213-2098-X

[9] ENGLE R F, GRANGER C J. Co- integration and correction: representation, estimation and testing $[\mathrm{J}]$. Journal of Econometric Society, 1987, 55 (2): 251- 276. http://dx.doi.org/10.2307/ 1913236

[10] Yue Changjun. The international comparison of the population of higher education [J]. Comparative education research, 2004, (2).

[11] Sun Shaorong, ZHu Junping. The international statistical analysis of the relationship between enrollment rate of higher education and GNP per capita [J]. University of Shanghai for Science and Technology,2000,3:247-251

[12] Huang Rongyuan. In the second half of the 20th century Chinese higher education scale fluctuation research-- the higher education 
development prediction at the beginning of the 21 st century [J]. Educational research, 2000, (10).

[13] Sun Shaorong, The international statistical research on the relationship between the higher education and the economic level [J]. China high education research, 2001, (4):30-34.

\section{AUTHORS}

Chao Ge, female, born in December, 1983. She obtained a Master degree in Law in 2009. At present, she works as section chief of Teaching Management Section in Humanities and Social Sciences College of Agricultural University of Hebei. Her title is Social Sciences Research Assistant. Her research field is Law. (e-mail: 68219008@qq.com).

Cun Li, male, born in February, 1982. He obtained a Master degree in Ideological and Political Education in 2008. He works as section chief of Market and Entrepreneurship Section in Career Education and Service Center for Students of Agricultural University of Hebei now. His title is lecturer. His research field is ideological and political education as well as management of students' employment and entrepreneurship (416711518@qq.com)

This work was supported in part by2015 Program supported by Social Sciences Fund of Agricultural University of Hebei: Study on Operation Strategies and Effectiveness of WeChat Career Accounts (Grant No. SK201534-4). Submitted 07 November 2015.Published as resubmitted by the authors 05 December 2015. 